\title{
A New Method for Sorting Radar Signal Based on Entropy Features
}

\author{
Jun Han, Xiaofei Lu, Minghao He and Xiaojie Tang ${ }^{*}$ \\ Air Force Early Warning Academy, Wuhan 430019, China \\ ${ }^{*}$ Corresponding author
}

\begin{abstract}
In order to figure out shortcomings of the existing method, such as the accuracy is not high and the method is sensitive to noise, we firstly Obtain the Wigner distribution space and bispectrum space for the received radar signal, then divide the space into subspace. Under the subspace distribution probability, the information entropy features of radar signals are constructed into two-dimensional eigenvectors, which reflects the energy distribution of the signal in these two spaces. Through the simulation results, it shows that extracted characteristic parameters have good separation and stability in the range of wide signal to noise ratio, verifying the validity of the method in this passage.
\end{abstract}

Keywords-component: sort; entropy feature; subspace; the Wigner distribution; bispectrum

\section{INTRODUCTION}

Radar source signal sorting is one of the key technologies of EW system ${ }^{[1]}$, referring to the separation of impulses belonging to different sources of impulses from the intercepted dense radar pulse stream. With the increasing proportion of new complex radar, the sort of unknown radar's source signal plays an increasingly important role. Current radar's source signal sort uses features mainly based on the analysis of diverse general parameters of the intercepted signal, such as $t_{T O A}, \theta_{A O A}, f_{R F}, \tau_{p w}$ and so on ${ }^{[2-6]}$. But these general parameters of sort features all have their drawbacks. When the $f_{R F}, \tau_{p w}$ and other parameters of the signal change fast and variously, the usability of these general sorting features will be greatly reduced. Intra-pulse characteristics are one of the most characteristic parameters of radar signals. Although some of the current radar signals vary widely in their conventional parameters, the characteristic parameters in the pulse have certain stability. At present, many scholars have applied the intra-pulse characteristic parameters such as wavelet features and complexity features to the classification and recognition of radar source signals, and achieved some success ${ }^{[7-9]}$.

Radar source signals can be viewed as a superposition of certain useful signals and random noise, which have a certain degree of uncertainty. This uncertainty is related to the signalto-noise ratio and the parameters of the signal received. In informatics, entropy represents the system's average uncertainty ${ }^{[10]}$. The information entropy in the signal refers to the average amount of information contained in the signal. It decreases as the prior probability increases, but increases with the posterior probability, reflecting the complexity of the information contained in the signal. When modulation form of radar source signal is certain, after a certain noise superimposed, it's information entropy maintain a certain level When the signal structure and transmission environment changes, the complexity of the information changes, uncertainty changes, and it's entropy changes as well for detection and reception system. Therefore, the information entropy can be used for radar radiation source signal sorting.

This paper starts from the Wigner distribution and double spectrum of radar source signal and extract the features of information entropy and a high accuracy sorting of unknown complex radar signals with low signal-to-noise ratio is achieved.

\section{INFORMATION ENTROPY}

Information entropy represents the measure of the overall information of the signal in an average sense. The formula is:

$$
H(S)=-\sum_{i=1}^{N} P_{i} \ln P_{i}
$$

In the formula, $X=\left\{x_{1}, x_{2}, \ldots, x_{N}\right\}$ is the discrete signal after sampling. From a wide area, it can be a signal on any domain, the probability of its magnitude is $P_{i}=P\left(x_{i}\right), i=1,2, \ldots, N$, and $\sum_{i=1}^{N} P_{i}=1$.

Radar radiation source signal amplitude is usually within a certain range of continuous distribution, and the amount of discrete signal after AD sampling is very large. According to the conventional method of obtaining information entropy, it is impossible to calculate the probability of the radar source signal according to the amplitude points one by one. Therefore, it is necessary to convert the radar source signal in the time domain to other domains in order to better obtain the entropy feature.

Information entropy can be established using a method of subspace distribution probabilities. Let $\mathrm{S}$ be the signal $\mathrm{X}$ 's feature space, $S_{1}, S_{2}, \ldots, S_{N}$ is its characteristic subspace, a complete division of $\mathrm{S}$. Then the distribution probability of signal $\mathrm{X}$ under the feature subspace $S_{N}$ can be expressed as follows: 


$$
P_{S_{i}}=\frac{\sum_{S_{i}} E\left(x_{i}\right)}{\sum_{S} E\left(x_{i}\right)}, i=1,2, \ldots, N
$$

Where $E(\cdot)$ is the energy function, $\mathrm{N}$ is the number of subspaces. From the formula we can see that $P_{S_{i}}$ is characterized by the proportion of the energy of the signal subspace $S_{i}$ occupied by the entire signal feature space.

According to the definition of information entropy, the information entropy of the signal under the probability of subspace distribution can be obtained:

$$
H_{S}(X)=-\sum_{i=1}^{N} P_{S_{i}} \ln P_{S_{i}}
$$

$H_{S}(X)$ characterizes the distribution of signal energy among subspaces of each feature. If the signal in a feature subspace mutation, the corresponding subspace probability will change, resulting in changes in information entropy. When each subspace energy is equal, the information entropy gets the maximum value, which is $H_{S}(X)=\ln N$, therefore, the standardization of information entropy can be expressed as:

$$
H_{S}(X)=\frac{-\sum_{i=1}^{N} P_{S_{i}} \ln P_{S_{i}}}{\ln N}
$$

\section{WIGNER DISTRIBUTION ENTROPY CHARACTERISTICS}

According to the definition in the previous section, the radar source signal can be converted to the corresponding spatial domain. The method of subspace distribution probability is used to establish the feature of information entropy.

For different modulation methods of radar signals, they have different time-domain distribution. Time domain analysis is a typical method of frequency domain feature analysis, which can establish the signal energy distribution status, widely used in the field of signal processing.

Wigner distribution is often used in time-frequency analysis of the method is a typical Cohen class time-frequency analysis. The Wigner distribution is defined as the Fourier transform of the central covariance function of the signal, which can fully reflect the instantaneous power spectral density characteristics of the signal in the energy space.

The Wigner distribution of the signal $s(t)$ is defined as:

$$
W_{x}(t, f)=\frac{1}{2 \pi} \int_{-\infty}^{\infty} x\left(t+\frac{1}{2} \tau\right) x^{*}\left(t-\frac{1}{2} \tau\right) e^{-j 2 \pi f \tau} d \tau
$$

Where, $x(t)$ is the analytical signal of $s(t), \tau$ is the time difference variable, $f$ is the frequency. The total integral of Wigner distribution in time-frequency and two-axis is equal to the signal energy $\mathrm{E}$, that is:

$$
\begin{aligned}
& \frac{1}{2 \pi} \int_{-\infty}^{\infty} \int_{-\infty}^{\infty} W_{x}(t, f) d t d f=\frac{1}{2 \pi} \int_{-\infty}^{\infty}|X(f)|^{2} d f=E \\
& \frac{1}{2 \pi} \int_{-\infty}^{\infty} \int_{-\infty}^{\infty} W_{x}(t, f) d t d f=\frac{1}{2 \pi} \int_{-\infty}^{\infty}|x(t)|^{2} d t=E
\end{aligned}
$$

From (5) to (7), we can see that the Wigner distribution has a clear physical meaning, which reflects the signal energy distribution in the time and frequency domains, and can fully describe the signal energy density distribution.

It can be seen from the above analysis that the different modulation forms of radar source signals have different energy distributions in each subspace of the Wigner distribution space, and the characteristics of the information entropy represented by them are also different.

Extraction of the Wigner distribution of signal characteristics of the signal entropy algorithm steps are as follows:

Step1 pre-process the received radar signal;

Step2 Find the Wigner spatial distribution of the radar signal according to formula (5)

Step3 subdivide the Wigner distribution space along the time axis and the frequency axis;

Step4 according to equation (2) to find the signal distribution probability in subspace;

Step5 obtains the information entropy feature of signal Wigner distribution according to formula (4);

\section{BisPECTRUM ENTROPY FEATURES}

Reconnaissance receiver receives the radar radiation source signal, after the pre- processing, the noise included includes various clutters, receiving system thermal noise and so on. Research shows that such as the weather by a large number of scattered.The spot-induced clutter and receive system thermal noise tend to Gaussian distribution. As a tool of time series analysis, high order spectrum can effectively suppress the influence of Gaussian noise. Therefore, the use of bispectrum analysis, can effectively suppress the purpose of Gaussian noise.

The power spectrum characterizes the distribution of signal energy over frequency and the bispectrum is the decomposition of signal skew in the frequency domain. The bispectrum defined by the high order cumulant is as follows: If the higher-order cumulants $c_{k x}\left(\tau_{1}, \ldots, \tau_{k-1}\right)$ of random sequences $\left\{x(n), x\left(n+\tau_{1}\right), \cdots, x\left(n+\tau_{k-1}\right)\right\}$ satisfy ${ }^{[9]}$. 


$$
\sum_{\tau_{1}=-\infty}^{\infty} \ldots \sum_{\tau_{k-1=-\infty}}^{\infty}\left|c_{k x}\left(\tau_{1}, \ldots, \tau_{k-1}\right)\right|<\infty
$$

Then the k-order spectrum is defined as the k-1 dimensional discrete Fourier transform of the k-order cumulant, that is:

$$
\begin{aligned}
& S_{k x}\left(\omega_{1}, \ldots, \omega_{k-1}\right)=\sum_{\tau_{1}=-\infty}^{\infty} \ldots \sum_{\tau_{k-1}=-\infty}^{\infty} c_{k x}\left(\tau_{1}, \ldots, \tau_{k-1}\right) \mathrm{e}^{-\mathrm{j}\left(\omega_{1} \tau_{1}+\ldots+\omega_{k-1} \tau_{k-1}\right)} \\
& \left|\omega_{i}\right| \leq \pi, \quad i=1, \cdots, k=1, \quad\left|\omega_{1}+\omega_{2}+\cdots+\omega_{k-1}\right| \leq \pi
\end{aligned}
$$

Bispectrum is third order spectrum, defined as:

$$
B_{x}\left(\omega_{1}, \omega_{2}\right)=\sum_{\tau_{1}=-\infty}^{\infty} \sum_{\tau_{2}=-\infty}^{\infty} c_{3 x}\left(\tau_{1}, \tau_{2}\right) \mathrm{e}^{-\mathrm{j}\left(\omega_{1} \tau_{1}+\omega_{2} \tau_{2}\right)}
$$

From the definition of bispectrum, the bispectrum reflects the energy distribution of the signal on the two-dimensional frequency axis. The energy distribution of the bispectrum space in different modulation signals is different, and the information entropy features are also different.

Extraction of radar signal bispectrum information entropy feature algorithm steps are as follows:

Step1 pre-process the received radar signal;

Step2 Find the bispectrum function space of radar signal according to (10);

Step3 subdivide the two frequency axes in the bispectrum function space;

Step4 according to equation (2) to find the signal distribution probability in subspace;

Step5 obtains the information entropy features of the signal spectral function space according to formula (4);

\section{SORTING STEPS}

Based on the above analysis, the received radar source signal is processed as follows to achieve sorting.

Step1 pre-process the received radar signal;

Step2 separately obtain the information entropy features of the Wigner distribution space of the radar signal, the information entropy features of the bispectrum function space;

Step3 using the extracted characteristic parameters based on KFCM algorithm to achieve sorting;

\section{Simulation Test}

\section{A. Simulation Conditions}

Eight types of radar source signals are simulated, with conventional modulated radar signal(CW), linear Frequency Modulated Radar Signal (LFM), frequency diversity radar signal (FD), frequency Coded Radar Signal (FSK), binary phase shift keying signal (BPSK), quadrature phase shift keying signal (QPSK), nonlinear FM radar signals (NLFM) and two-phase encoded hybrid chirp signal (BPSK + LFM) as an example. The frequencies of the frequency diversity radar signals are $10 \mathrm{MHz}$ and $50 \mathrm{MHz}$. The frequencies of the frequency-coded radar signals are $20 \mathrm{MHz}$ and $40 \mathrm{MHz}$. The other types of radar signals have a carrier frequency of $30 \mathrm{MHz}$. The pulse width of all the signals is $10 \mu$ s and the sampling frequency is $120 \mathrm{MHz}$. The frequency bandwidth of LFM and NLFM signals are $2 \mathrm{MHz}$ and $5 \mathrm{MHz}$ respectively. The phase encoding rule of BPSK signal is [1001011001], and the phase encoding rule of QPSK signal is [0123031311]. When the signal-to-noise ratios are $5 \mathrm{~dB}, 10 \mathrm{~dB}, 15 \mathrm{~dB}$ and $20 \mathrm{~dB}$, each type of signal produces 200 samples and 1024 samples for each sample.

\section{B. Experimental Analysis}

When the signal-to-noise ratios are $5 \mathrm{~dB}, 10 \mathrm{~dB}, 15 \mathrm{~dB}$ and $20 \mathrm{~dB}$, each type of signal produces 200 samples and 1024 samples for each sample. The information entropy features of the Wigner distribution space and the information entropy features of the bispectrum function space of each type of signal at the corresponding signal-to-noise ratio are respectively obtained respectively, denoted by $C_{w v}$ and $C_{s p}$ respectively. The average and variance of $C_{w v}$ and $C_{s p}$ for each type of signal for each signal-to-noise ratio are averaged and the results are shown in Figure 1 and Figure 2.

$1 \sim 8$ in the figure are the above-mentioned 8 kinds of signals respectively. As can be seen from Figure 1, the $C_{w v}$ and $C_{s p}$ eigenvalues of Type 2 and Type 8 signals are relatively close at $5 \mathrm{~dB}$ signal-to-noise ratio. As can be seen from the detail diagram in the figure, there is a partial overlap between the two types of signals. The other types of signals do not have the two eigenvalues that are close to each other, so combining these two types of features can separate the remaining six types signal. When the signal to noise ratio reaches $20 \mathrm{~dB}, 8$ types of signals can be well separated.
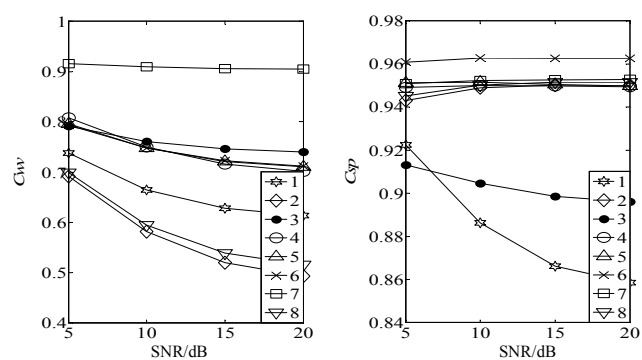

FIGURE I. MEANS OF $C_{W V}$ AND CSP AT DIFFERENT SNR 


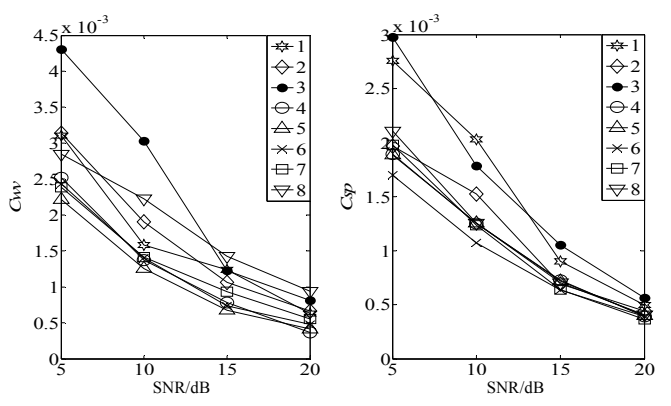

FIGURE II. VARIANCES OF $C_{W V}$ AND CSP AT DIFFERENT SNR

KFCM algorithm is used to sort 8 types of radar source signals. The characteristic parameters used for sorting are $C_{w v}$ and $C_{s p}$, the initial clustering number $c=2$, the maximum possible number of categories $c_{\max }=8$, the iteration number $\mathrm{T}$ is set as 100 , the stopping condition $\varepsilon \leq 0.00001$, the kernel function is Gaussian radial basis nuclear.

Under different signal-to-noise ratios, each 100 montecarlo experiments were performed. The average sorting accuracy of 8 types of radar source signals is shown in Table 1 . It can be seen from the table that in a wide range of signal-tonoise ratio, the sorting accuracy of $C_{w v}$ and $C_{s p}$ characteristic parameters is very high. When the signal-to-noise ratio reaches $15 \mathrm{~dB}$, the accuracy of sorting the signal of 8 types of radar source is $100 \%$. As the SNR decreases, the accuracy of sorting decreases slightly. When SNR is $5 \mathrm{~dB}$, the characteristic parameters of LFM radar and BPSK + LFM radar partially overlap, so the accuracy of sorting fails to reach $100 \%$, but the average sorting accuracy is above $98 \%$; The remaining six types of radar source signal does not overlap, sorting accuracy rate of $100 \%$.

TABLE I. SORT RATES OF 8 CLASSES RADARS

\begin{tabular}{|c|c|c|c|c|c|c|c|c|c|}
\hline SNR & CW & LFM & FD & FSK & BPSK & QPSK & NLFM & BPSK+ LFM & $\begin{array}{c}\text { General Sorting } \\
\text { Accuracy }\end{array}$ \\
\hline $5 \mathrm{~dB}$ & 100 & 94.7 & 100 & 100 & 100 & 100 & 100 & 96.3 & 98.875 \\
\hline $10 \mathrm{~dB}$ & 100 & 98.6 & 100 & 100 & 100 & 100 & 100 & 99 & 99.7 \\
\hline $15 \mathrm{~dB}$ & 100 & 100 & 100 & 100 & 100 & 100 & 100 & 100 & 100 \\
\hline $20 \mathrm{~dB}$ & 100 & 100 & 100 & 100 & 100 & 100 & 100 & 100 & 100 \\
\hline
\end{tabular}

In this paper, the signal of radar source is preprocessed firstly, the Wigner distribution space and bispectrum space of the signal are obtained, then the subspace is divided, the information entropy characteristics of the signal in these two feature spaces are obtained, Radiation source signal sorting. Through the comparison of simulation experiment and method, this characteristic parameter reflects the good inter-class separation and stability under the condition of wide signal-tonoise ratio, which is of certain reference value.

\section{ACKNOWLEDGEMENT}

This work is supported by natural of Hubei province (no.2016CFB288)

\section{REFERENCES}

[1] Wiley R G. ELINT: The Interception and Analysis of Radar Signals [M]. Second Edition, Boston, MA: Artech House, 2006: 317-334.

[2] NELSON D J . Special purpose correlation functions for Improved signal detection and parameter estimation[C]//ICASSP , 1993 , $4: 73276$

[3] Li He-Sheng, Han Yu, Cai Ying-wu. Overview of the crucial technology research for radar signal sorting [J]. Systems Engineering and Electronics, 2005,27(12):2035-2040.

[4] Chen Sheng, Wang Xing, Chu Wei, Zhang Guan-rong. Research on recognition of phased-array radar based on analysis of pulses amplitude [J]. Computer Engineering and Applications, 2012,48(23):120-124.

[5] Wang Shi-qiang, Zhang Deng-fu. Novel radar signal sorting method with low complexity [J]. Journal of XIDIAN University,2011,38(4):148153.

[6] Gee, Wesley A. Radar warning receiver (RWR)time-coincident pulse data extraction and processing[C]. IEEE National Radar Conference Proceedings, Atlanta, United states.2012: 752-757.

[7] Ren Ming-qiu, Huo Jin-yan. Radar emitter recognition based on wavelet ridge and FSVM [J]. Chinese Journal of Scientific Instrument, 2010,31(6): 1424-1428.
[8] Chen Tao-wei,Xin Ming. Feature extraction based on wavelet transform for radar emitter signals [J]. Information and Electronic Engineering, $2010,8(4): 436-440$.

[9] ZHANG Gexiang, JIN Weidong, HU Laizhao. Radaremitter signal recognition based on complexity features $[\mathrm{J}]$. Journal of Southwest Jiaotong University(EnglishEdition), 2004,12 (2) : 1162122.

[10] Shannon C E. A mathematical theory of communications [J]. Bell System Technical Journal, 1948, 27:379-42.

[11] Havens, Timothy C. Cluster validity for kernel fuzzy clustering[C]. IEEE International Conference on Fuzzy Systems, Brisbane, Australia.2012: 124-127.

[12] Miyamoto, Sadaaki. Different objective functions in fuzzyc-means algorithms and kernel-based clustering [J]. International Journal of FuzzySystems, 2011,13(2):89-97. 